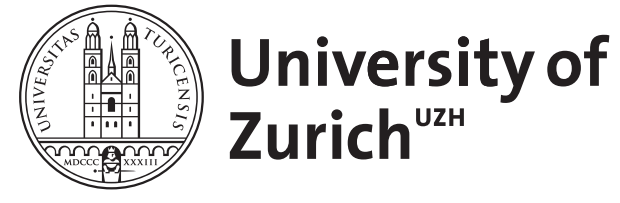

\title{
Ringen um Selbstbestimmung
}

Budnik, Christian

DOI: https://doi.org/10.1515/dzph-2018-0019

Posted at the Zurich Open Repository and Archive, University of Zurich ZORA URL: https://doi.org/10.5167/uzh-187275

Journal Article

Published Version

Originally published at:

Budnik, Christian (2018). Ringen um Selbstbestimmung. Deutsche Zeitschrift für Philosophie, 66(2):243250.

DOI: https://doi.org/10.1515/dzph-2018-0019 


\section{Buchkritik}

\section{Christian Budnik* \\ Ringen um Selbstbestimmung}

https://doi.org/10.1515/dzph-2018-0019

Beate Rössler. Autonomie. Ein Versuch über das gelungene Leben. Berlin, Suhrkamp, 2017, 443 S.

Nur die wenigsten Personen würden von sich behaupten, dass sie nicht autonom sind. Gleichzeitig fällt es uns nicht schwer, Faktoren auszumachen, die Autonomie untergraben oder unmöglich machen. Nur zu gut kennen wir Fälle, in denen Personen nicht wissen, was sie eigentlich wollen, oder Dinge tun, die sie eigentlich nicht wollen, etwa weil sie sich über sich selbst täuschen oder manipuliert worden sind. Autonomie ist ein zentraler Wert, und gleichzeitig ist sie ein ständig bedrohter Wert in unserem Leben. Es ist genau diese Spannung zwischen unserem Selbstverständnis als autonome Akteurinnen und Akteure und der alltäglichen Erfahrung des Scheiterns von Autonomie, die das Grundthema des Buches von Beate Rössler darstellt. Die zentrale Botschaft Rösslers lautet, dass Autonomie möglich ist, dass wir aber nur dann ein angemessenes Verständnis von Autonomie erreichen, wenn wir die Spannungen und Widersprüche des gelebten Alltags autonomer Akteurinnen und Akteure nicht aus dem Blick verlieren. Weite Teile des Buches beschäftigen sich entsprechend mit der Frage, mit welchen Problemen um Autonomie bemühte Personen konfrontiert sind und was uns diese Probleme im Hinblick auf Autonomie lehren können.

Den Beginn macht Rössler hierbei mit dem Phänomen der Ambivalenz, indem sie gegen die Auffassung von Harry Frankfurt argumentiert, nach der Ambivalenz eine ,Krankheit des Willens' darstellt und über die Externalisierung einer der an einem Ambivalenzkonflikt beteiligten Optionen aufgelöst werden muss (Kap. 2). Sie macht in diesem Zusammenhang darauf aufmerksam, dass es möglich ist, auf authentische Weise ambivalent zu sein, und erläutert diese These anhand von Identitätskonflikten, bei denen es Personen gelingen kann, durch geschickte Identitätswechsel ihre Autonomie aufrechtzuerhalten. Als um Autonomie bemühten Personen könne es uns letztlich nicht darum gehen, Ambivalenzen auszumerzen, so Rössler, sondern eher darum, einen souveränen Umgang mit ihnen zu entwickeln.

*Kontakt: Christian Budnik; christian.budnik@philo.unibe.ch 
Als mit Autonomie unvereinbar werden dagegen jene Phänomene ausgewiesen, von denen die Selbsterkenntnis handelnder Personen unterminiert wird (Kap. 4). Rössler diskutiert hier auf aufschlussreiche Weise das Phänomen der Selbsttäuschung und argumentiert, dass wir nur dann als selbstbestimmt betrachtet werden können, wenn wir wissen, was wir denken und wollen bzw. wer wir sind oder sein möchten. Im Rahmen der Diskussion von Faktoren, die Selbsterkenntnis bedrohen können, macht Rössler auf Fälle aufmerksam, in denen die Selbstachtung von Personen untergraben wird, so dass sie aufhören, sich als Subjekte zu verstehen, die in der Lage sind, auf angemessene Weise mit Gründen zu operieren. Auf diese Weise etabliert sie Selbstachtung als eine wichtige Bedingung für Autonomie und bereitet den Boden für ein soziales Verständnis der Bedingungen von Autonomie.

Besonders spannend sind Rösslers Überlegungen zu der Frage, inwiefern Bedrohungen der individuellen Privatheit von Personen als Gefahren für ihre Autonomie verstanden werden können (Kap. 7). Bezüglich informationeller Privatheit argumentiert sie hierbei, dass sie insofern zentral für Autonomie ist, als sie eine Voraussetzung dafür darstellt, dass wir uns einerseits in unterschiedlichen Kontexten auf unterschiedliche Weise inszenieren und entsprechend handeln können, andererseits auf angemessene Weise an persönlichen Beziehungen teilnehmen können. Inwiefern Autonomie durch ein Aushöhlen von Beziehungen bedroht wird, diskutiert Rössler hierbei unter Rekurs auf die ,Abschaffung des Privaten“ in der Welt der sozialen Medien. Dass der Schutz des Privaten aber durchaus auch problematisch für die Autonomie von Personen sein kann, macht sie plausibel, indem sie die Auffassung diskutiert, dass die traditionelle Unterscheidung zwischen einem öffentlichen Lebensbereich und einem Raum häuslicher Privatheit die Unterdrückung der Autonomie von Frauen befördert hat.

Als zentral für Rösslers Vorhaben muss die Argumentation aufgefasst werden, in deren Rahmen sie dafür plädiert, dass Autonomie und ihre Bedingungen nicht ohne den sozialen Kontext des Lebens von Personen zu verstehen sind, und gleichzeitig darauf aufmerksam macht, dass sich eine besonders verbreitete Gefahr für Autonomie gerade aus der Tatsache ergibt, dass um Autonomie bemühte Akteurinnen und Akteure immer auch als sozial eingebettete Wesen zu betrachten sind (Kap. 8). Im Hinblick auf Letzteres konzentriert sich Rösslers Diskussion zunächst auf das Phänomen der adaptierten Präferenzen, bevor sie auf die Problematik des gleichen Zugangs zu wertvollen Optionen in liberaldemokratischen Gesellschaften und schließlich auf die Bedrohung von Autonomie in Gesellschaften, die schlicht unterdrückend sind, zu sprechen kommt. Im Hinblick auf eine positive Bestimmung von Autonomie grenzt sich Rössler zunächst kritisch von prozedural-individualistischen Theorien ab. Ein relationaler Ansatz, wie etwa von Marina Oshana vertreten, ist in ihren Augen allerdings 
zu anspruchsvoll, weil er implizieren würde, dass „nur noch wenige Personen, auch in liberal-demokratischen Gesellschaften, überhaupt autonom genannt werden können“ (333). In der Folge schließt Rössler deshalb an ein weniger substantielles Verständnis der sozialen Bedingungen von Autonomie an, indem sie dafür argumentiert, dass eine autonome Person in der Lage sein muss, sich als „Autorität über die eigenen Gründe“ (335) zu sehen und eigenständige Projekte zu verfolgen, wozu sie allerdings auf die Anerkennung durch andere Personen angewiesen ist.

Autonomie ist also auf mannigfache Weise in Gefahr, sei es durch individuelle Verzerrungen oder durch soziale Störfaktoren, denen wir nie ganz aus dem Weg gehen können. Warum klammern Personen sich angesichts dieser Schwierigkeiten an Autonomie? Was macht Autonomie so wertvoll für uns? Im Verlaufe ihrer Argumentation gibt Rössler eine Reihe von Antworten auf diese Fragen (vgl. etwa 15 u. 29). Es sind aber vor allem ihre Ausführungen in Kap. 3 und Kap. 6, die in diesem Kontext aufschlussreich sind. Hier wird nichts weniger als der Versuch unternommen, eine Theorie des gelungenen Lebens zu entwickeln, indem auf die Frage nach dem Sinn des Lebens sowie jene nach dem guten Leben eingegangen und gezeigt wird, inwiefern beide zum gelungenen Leben beitragen und Autonomie zur Voraussetzung haben. In diesem Zusammenhang argumentiert Rössler einerseits gegen ein subjektivistisches und auf Wunscherfüllung fokussiertes Verständnis des sinnvollen Lebens (und von Autonomie); andererseits verteidigt sie eine „schwach paternalistische“ (vgl. 232-233) Theorie des guten Lebens, die Autonomie als zentrales Gut auszeichnet, ohne dabei substantielle Annahmen im Hinblick auf bestimmte Ziele oder Projekte zu machen.

Bereits diese zwangsläufig selektive Rekonstruktion der wichtigsten Argumentationsziele von Rössler deutet darauf hin, dass ihr Buch einen sehr großen Bogen zu spannen versucht. Dass dies nicht gelingen kann, ohne über die Details der verschiedenen philosophischen Debatten, die hier thematisiert werden, hinwegzusehen, versteht sich von selbst. Zudem soll das Buch explizit nicht als ,wissenschaftliche Abhandlung im strengen Sinn“ (11) verstanden werden, und es richtet sich auch an ein mit Philosophie unvertrautes Publikum. Rössler gelingt es hierbei auf ganz vorzügliche Weise, genuin philosophische Fragestellungen so zu formulieren, dass sie als unmittelbar relevant und ,lebensnah' erscheinen. Zudem thematisiert sie zusätzlich zu den aus philosophischen Debatten bekannten Fragestellungen originelle neue Aspekte; ich denke hier etwa an ihre Ausführungen zur Quantified-Self-Bewegung (Kap. 4) oder zur Praxis des Tagebuchschreibens als einer Form des Ringens um Selbstbestimmung (Kap. 5). Gleichzeitig handelt es sich bei dem Buch aber keinesfalls nur um ,Philosophie für Laien': Wie angedeutet, vertritt Rössler in den einzelnen Kapiteln eigene Positionen im Hinblick auf systematische Fragestellungen, und sie verteidigt im Rahmen des Buches 
nicht zuletzt auch eine spezifische externalistische Autonomietheorie. Dies alles böte mehr als genügend Stoff zu Diskussionen und kritischen Nachfragen, die einzelne Thesen und Argumentationslinien der Ausführungen von Rössler betreffen. Im Gegensatz dazu möchte mich an dieser Stelle auf die Erwähnung von zwei allgemeinen Problemen beschränken, mit denen die Argumentation von Rössler konfrontiert sein könnte, und die im weitesten Sinn den methodologischen Status ihres Vorgehens betreffen.

(i) Eines der großen Ziele des Buches besteht darin, genau zu untersuchen, auf welche Weisen Autonomie im alltäglichen Leben von Personen auf die Probe gestellt oder bedroht sein kann. Man könnte meinen, dass man dazu zunächst einen wenigstens vorläufigen Begriff von Autonomie entwickeln müsste, um in den angesprochenen Szenarien überhaupt erst Instanzen der Bedrohung von Autonomie individuieren zu können. Rössler möchte diesen Weg explizit nicht beschreiten. In einem informativen ersten Kapitel wird zwar der Versuch einer begrifflichen Annäherung an Autonomie unternommen, die die Leserinnen und Leser von Kants Autonomiebegriff über eine Bestimmung des Verhältnisses von Autonomie und Freiheit bis hin zu modernen Autonomietheorien lotst; im Wesentlichen soll sich aber eine Theorie persönlicher Autonomie in dem Buch „unter der Hand“ (27) entwickeln, während Rössler die verschiedenen Möglichkeiten des alltäglichen Scheiterns von Autonomie nacheinander in den Blick nimmt.

Prinzipiell ist gegen so ein Vorgehen nichts zu sagen, es fragt sich allerdings, ob man diese methodische Haltung nicht noch etwas genauer hätte auf den Punkt hätte bringen können. Was soll es denn heißen, dass sich eine Theorie „unter der Hand“ entwickeln wird? Plausibel scheint mir in dieser Hinsicht eine Antwort, die darauf aufmerksam macht, dass es zu den Adäquatheitskriterien für eine Autonomietheorie gehört, dass sie Autonomie auch angesichts der alltäglichen Herausforderungen, mit denen um Autonomie bemühte Akteurinnen und Akteure konfrontiert sind, als möglich ausweist. Genau dies scheint auch das große Konditional zu sein, unter dem Rösslers Buch zu lesen ist: Wenn wir davon ausgehen, dass es (in der Regel oder für die meisten von uns) möglich ist, ein gelungenes (und ergo sinnvolles) Leben zu führen, und wenn es wahr ist, dass Autonomie eine notwendige Bedingung eines solchen Lebens ist, dann muss Autonomie so konstruiert werden, dass die bloße Tatsache, dass unser ,gelebter Alltag` das Erreichen von Autonomie problematisch macht, nicht automatisch darauf hinausläuft, dass Autonomie unmöglich ist.

Die große Frage ist selbstverständlich, ob die Tatsache, dass wir in unserem Bestreben, ein autonomes Leben zu führen, mit Hindernissen im Alltag konfrontiert sind, überhaupt irgendeinen Einfluss auf die begriffliche Bestimmung von Autonomie haben kann. Rössler scheint dieser Auffassung zu sein, indem sie 
ihr Autonomieverständnis immer wieder von einem ,idealen` Autonomiebegriff abgrenzt, so etwa an der folgenden Stelle: „Denn auch wenn ein idealer Begriff von Autonomie - und ein vollkommen autonomes, ideales Leben - vielleicht keine jener Widersprüchlichkeiten und Hindernisse enthielte, so sind diese gerade kennzeichnend für einen Begriff und ein Leben, die dem nichtperfekten Alltag und dem Streben nach dem selbstbestimmten und gelungenen Leben gerecht werden“ (396, Hervorh. im Orig.). Es ist aber eine Sache zu behaupten, dass es Personen möglich ist, ein in jeder Hinsicht autonomes Leben zu führen, und eine ganz andere, eine Begriffsbestimmung von Autonomie vorzulegen, die zunächst keine Rücksicht darauf nimmt, dass es Personen manchmal (und aus verschiedenen Gründen) nicht einfach fällt, autonom zu sein. Dass man sich nicht recht vorstellen kann, was es heißen soll, dass ein Begriff Widersprüchlichkeiten und Hindernisse ,enthalten' soll, wie es das obige Zitat nahelegt, deutet bereits darauf hin, dass wir möglicherweise keine Alternative $\mathrm{zu}$ einem solchen Vorgehen haben. (Zudem könnte ein ,idealer‘ Autonomiebegriff durchaus auch eine Orientierungsfunktion haben, auch wenn er im Alltag niemals vollständig realisiert werden könnte.)

Anhand von Rösslers Rekonstruktion des Verhältnisses von Ambivalenz und Autonomie lässt sich dieser letzte Punkt etwas konkreter formulieren: Rössler hat sicher Recht, wenn sie gegen Frankfurt argumentiert, dass es keine gute Idee ist, Ambivalenzkonflikte aufzulösen, indem man einen Teil des Konfliktes externalisiert oder unterdrückt. Sie macht auch auf überzeugende Weise plausibel, dass die Identitäten von Akteurinnen und Akteuren komplex und brüchig sind und dass sich daraus verschiedene alternative Formen des Umgangs mit Ambivalenzkonflikten ergeben (vgl. 79 ff.) Dies alles läuft aber nur auf eine Kritik an Frankfurts Vorschlag hinaus, wie man mit Ambivalenz umgehen sollte, und stellt nicht unbedingt eine Kritik an der Auffassung dar, dass Ambivalenz eine ,Krankheit des Willens‘ ist. Die Tatsache, dass Rössler auf eine phänomenologisch komplexe Weise Ambivalenzkonflikte in den Blick nimmt, hat demnach keine Auswirkung auf die (begriffliche) These, nach der Ambivalenz in einem Spannungsverhältnis zu Autonomie steht, sondern sie öffnet uns die Augen dafür, wie unterschiedlich Ambivalenzkonflikte sein können und wie viele Optionen uns offenstehen, wenn wir angesichts solcher Konflikte unsere Autonomie erhalten wollen. Dies gilt auch für die Identitätskonflikte, die Rössler $u$. a. anhand des Beispiels von Maria Lugonez diskutiert, die beständig zwischen der homophoben Kultur ihrer Familie und der Kultur ihres homosexuellen Freundeskreises wechselt und auf diese Weise einen souveränen Umgang mit ihrer Ambivalenz an den Tag legt (vgl. 88 ff.). Wiederum zeigt dieses Beispiel, dass es möglich ist, angesichts von Ambivalenz autonom zu bleiben, aber es deutet eben auch darauf hin, dass es im Hinblick auf Autonomie noch besser wäre, wenn man gar nicht erst ambivalent 
wäre: Wenn Maria Lugonez als autonome Akteurin einen Wunsch frei hätte, dann würde sie sich wahrscheinlich nicht wünschen, besonders souverän zwischen ihren Identitäten als Latina und Homosexuelle wechseln zu können, sondern sie hätte eher den Wunsch, die Kultur ihrer Familie möge toleranter im Hinblick auf Homosexualität werden.

(ii) Um die Spannungen zwischen unserem Selbstverständnis als autonome Akteurinnen und Akteure und unseren alltäglichen Erfahrungen des Scheiterns von Autonomie in den Blick zu bekommen, verwendet Rössler immer wieder literarische Beispiele. Ihre Begründung dafür ist, dass „der gelebte autonome - oder gerade nicht autonome - Alltag seine ganz eigene Phänomenologie und Plausibilität hat“ und „Schriftstellerinnen und Schriftsteller [...] dies zumeist besser beschreiben [können] als selbstgestrickte - manchmal recht unbeholfene - philosophische Beispiele“ (21). Auch in diesem Zusammenhang wäre es hilfreich, noch mehr zu der Frage nach dem methodologischen Status, der solchen Beispielen in der Argumentation von Rössler zukommt, zu erfahren. Manche der Beispiele, die von Philosophinnen und Philosophen ,gestrickt' werden, haben eben absichtlich einen ganz anderen Charakter als literarische Beispiele, nämlich immer dann, wenn sie als Gedankenexperimente formuliert werden: Hier ist es gerade ein Vorzug und kein Nachteil eines Beispiels, wenn es keine reiche Phänomenologie enthält und wenn es von vielen Gesichtspunkten abstrahiert, weil dadurch gewissermaßen kontrollierte Bedingungen für die Diskussion einer bestimmten These geschaffen werden. Es scheint allerdings klar zu sein, dass die Beispiele aus der Literatur, die Rössler verwendet, nicht die Funktion von Gedankenexperimenten erfüllen sollen. Welche Rolle spielen sie stattdessen?

Ihre Funktion in Rösslers Argumentation erschöpft sich offenbar nicht darin, etwas zu illustrieren. An manchen Stellen sollen die Beispiele vielmehr eine bestimmte These plausibilisieren helfen, und meistens handelt es sich dabei um Varianten der zentralen These von der Grundspannung zwischen Autonomie und heteronomen Aspekten unseres Alltags. Eine Gefahr, die mit einem solchen Vorgehen verbunden ist, spiegelt den Punkt, mit dem ich diese kritische Diskussion unter (i) begonnen habe: Wir haben nur ein sehr vages vortheoretisches Verständnis von Autonomie, und es ist nicht klar, wie wir Instanzen von autonomem oder heteronomem Verhalten in einem literarischen Werk als solche identifizieren sollten, ohne schon einen bestimmten Autonomiebegriff vorauszusetzen.

Ein damit verwandtes Problem besteht darin, dass die Frage, ob und inwiefern eine Person autonom ist - zumindest, wenn man diesen Begriff so umfassend verstehen möchte, wie Rössler es tut -, eine hochkomplexe Angelegenheit darstellt, für die man eben mehr als nur einen Ausschnitt aus dem Leben der betreffenden Person kennen müsste. Eben deshalb lenkt ja Rössler den Blick auf literarische Beispiele, könnte man an dieser Stelle entgegnen. Allerdings wird dadurch nicht 
immer der gewünschte Effekt erzielt. Manchmal funktioniert der Verweis auf eine literarische Figur wie der Verweis auf eine Art gemeinsamen Freund, dem gegenüber man nicht zur Verschwiegenheit verpflichtet ist und dessen Erfahrungen herangezogen werden können, um Thesen, für die man ansonsten empirische Belege vorweisen müsste, zu illustrieren. Dies ist der Grund dafür, weshalb in solchen Zusammenhängen auf kanonisierte Werke der Literatur Bezug genommen wird, bei denen vorausgesetzt werden kann, dass alle Leserinnen und Leser mit den jeweiligen Figuren gut vertraut sind: Es reicht dann ein Hinweis auf die Zwickmühle, in der sich Antigone oder Hamlet, Elisabeth Bennet oder Raskolnikow befinden, und es steht uns sozusagen ein ganzes Leben vor Augen, aus dem sich unter Umständen etwas für eine philosophische Fragestellung lernen lässt. Wann immer Rössler sich auf solche weithin bekannten Figuren bezieht oder sich genügend Raum nimmt, um weniger bekannte Figuren zu charakterisieren, gelingen ihre Versuche, eine bestimmte Behauptung zu plausibilisieren und bereichern die Lektüre und das Verständnis ihrer Ausführungen auf eine ganz besondere Weise.

An anderen Stellen ist dagegen die Funktion der Beispiele, die Rössler anführt, weniger klar. Im Rahmen der Diskussion des Phänomens der Entfremdung im sechsten Kapitel heißt es etwa: „Mein erstes Beispiel soll zeigen, was es heißt, dass das eigene Leben nicht mehr als selbstbestimmt, als eigenes empfunden werden kann, und warum es überhaupt sinnvoll ist, hier als Gegenbegriff auf den Begriff der Entfremdung zu verweisen“ (259). Es folgt eine kurze Beschreibung von Eilis, der Protagonistin des Romans Brooklyn von Colm Tóibín, die aus Irland nach Brooklyn aufbricht, um dort Arbeit zu finden. Danach zitiert Rössler eine Passage aus dem Roman, in der ein personaler Erzähler davon berichtet, wie wenig sich die neue Umgebung, in der Eilis ihren Alltag bestreiten soll, wie „ein Teil von ihr“ anfühlt, wie „unecht“ und „leer“ (260) sie im Vergleich zu der Welt ist, die sie verlassen hat. Dass die Situation etwas mit Entfremdung zu tun hat, könnte man Rössler an dieser Stelle ,schenken', auch wenn man sich dabei die Frage stellt, ob jedes Erlebnis einer unvertrauten Umgebung automatisch als ein Fall von Entfremdung zählen sollte. Weitaus problematischer ist, dass man - zumindest ohne die Kenntnis weiterer Details aus dem Leben von Eilis - nicht verstehen kann, inwiefern die Situation als ein Fall zu betrachten ist, in dem eine Person das eigene Leben nicht als selbstbestimmt empfindet. Wo zweifelt Eilis denn genau an ihrer Autonomie? Sollen wir als Leserinnen und Leser des Buchs von Rössler diesen Zweifel aus der Situation herausdestillieren, indem wir uns empathisch in sie hineinversetzen? Dazu ist die zitierte Passage aber zu kurz: Was ein ganzer Roman leistet, lässt sich nicht auf acht Sätze daraus reduzieren. Oder sollen wir Rössler aufs Wort glauben, dass wir es hier mit Heteronomie im Alltag zu tun haben? Aber dann braucht es den Hinweis auf den Roman im Grunde nicht mehr. 
Bei all dem handelt es sich um vereinzelte Probleme, die kaum den Wert dieses Buches als Ganzes schmälern. Wer es liest, wird mit großer Wahrscheinlichkeit anfangen, etwas anders als gewohnt über Autonomie nachzudenken. Dies ist zum großen Teil der Tatsache geschuldet, dass Rössler eben diese und keine anderen methodischen Entscheidungen trifft. Es bleibt demnach dabei, dass die Fülle des (durchaus auch literarisch vermittelten) Lebens, der man auf jeder Seite des Buches begegnet, seine Lektüre insgesamt zu einer Bereicherung macht und bekannt geglaubte philosophische Fragestellungen auf eine interessante und für weitere Diskussionen fruchtbare Weise in ein neues Licht rückt. 